\title{
Adaptive Transformer Inrush Current Identification Principle Based on Second Harmonic
}

\author{
Xinyu Wang ${ }^{1}$, Bin Tian ${ }^{1,2}$, Liqun $\mathrm{Wu}^{1}$, Ling Wang ${ }^{1}$ and Hong Tan ${ }^{1}$ \\ ${ }^{1}$ College of Electrical Engineering and New Energy, China Three Gorges University, Yichang, China,443002 \\ ${ }^{2}$ Enshi power supply company, State grid Hubei electric power company, Enshi, China, 445000
}

\begin{abstract}
In order to prevent the maloperation of the transformer differential protection device affected by transformer magnetizing inrush current and internal short circuit fault current, the device should be able to identify the internal fault current and inrush current. Accordingly, this paper is aimed at adaptive transformer inrush current identification based on the second harmonic. The main idea is to introduce the floating threshold value on the basis of the traditional second harmonic brake and to track the change characteristics of the content of second harmonic and fundamental wave in real time. This method not only inherits the advantage of the original second harmonic braking method, but also helps to identify the internal fault and inrush current quickly, and improve the sensitivity of the magnetizing inrush current.
\end{abstract}

Keywords-magnetizing inrush; transformer protection; second harmonic; differential protection

\section{INTRODUCTION}

With the modern power grid transmission voltage increasing, the applications of large capacity transformer have been used widely and higher requirements on the transformer protection reliability and speed have been put forward. Either conventional protection or microcomputer protection, identification of inrush current has been a key problem in transformer differential protection. Due to the shunt capacitor of reactive power compensation and the distribution capacitance of EHV transmission line, there is large magnetizing inrush current when the transformer internal fault occurs.

Differential protection is the main protection of transformer internal fault. The differential current flowing through the differential protection device may be the inrush current generated by the internal fault current of the transformer and the voltage recovery when the transformer is unloaded or the external fault is removed. The magnetizing inrush current is as large as the short circuit current. If some measures are not used to avoid the inrush current, the transformer differential protection device is likely to malfunction.

This paper expounds the inrush current of transformer and its waveform characteristics and modeling simulation analysis by simulation of three-phase transformer on the condition of no-load closing and removing external fault. A second harmonic adaptive transformer inrush current identification principle is proposed. This principle introduces the floating threshold value on the basis of the traditional second harmonic brake and to track the change characteristics of the content of second harmonic and fundamental wave in real time. This method not only inherits the advantage of the original second harmonic braking method, but also helps to identify the internal fault and inrush current quickly, and improve the sensitivity of the magnetizing inrush current.

\section{MAGNETIZING INRUSH}

One side of the transformer voltage increasing suddenly, voltage variation and the remanence in-phase stacking makes transformer core saturation and transformer excitation inrush occur. When transformer no-loaded closes or external failure resects, the corresponding voltages increase at once, and both cases are likely to produce a lot of excitation inrush current. The specific process is as follows: under the condition of stable operation, the magnetic flux phase of transformer core should be 90 degrees behind the applied voltage. If no-load closing, the circuit is connected while the instantaneous voltage is zero, in that way core flux is negative. Since the flux cannot change instantly, there may be a non-periodic positive flux component, and after half a cycle the flux reaches $2 \mathrm{~Wb}$. At this point, if there are traces of residual magnetic of the iron core, the total flux may be $2 \mathrm{~Wb}$ plus a small amount of magnetic flux, which leads to transformer core saturation and sharp increase of excitation current (the maximum value can reach 6 to 8 times the rated current).

When transformer no-load closes, excitation inrush current only appears on the high-voltage side and produces very big differential current. Furthermore, the large excitation inrush current amplitude is easy to cause the differential protection maloperation.

\section{CHARACTERISTICS OF MAGNETIZING INRUSH}

The waveforms of the internal fault current and inrush current are obtained respectively by simulation. After comparing two kinds of waveforms, the following characteristics of inrush current are drawn.

- Due to the large non-periodic component of inrush current waveform, at the first few cycles the current is inclined to the time axis side and decays rapidly. After 0.5 seconds, the value is less than 0.25 to 0.5 times the rated current. In another word, the waveform is completely biased to the positive time axis at the first 2 cycles, and the amplitude decreases rapidly.

- In the first few cycles, the inrush current is discontinuous. In general, there is a dead angle between the two waveforms, which is corresponding to the normal working range of the iron core. The dead 
angle ranges from 120 degrees to 180 degrees in each cycle, while the minimum value is not less than 80 degrees to 100 degrees.

- There are a large number of harmonic components in the inrush current waveform, including the majority of the second harmonic component and the minority of fourth or higher harmonic.

\section{INRUSH CURRENT IDENTIFICATION PRINCIPLE}

The process of the traditional second harmonic braking is divided into two stages. The first cycle after the transformer no-load closing is the first stage, during which time the maximum ratio of harmonics brake is adopted with OR gate brake based on traditional three-phase harmonic. On account of the rapid increase of the content of 2 nd harmonic at the first two cycles after switching on, the theory of 2 nd harmonic restraint is able to fully guarantee differential protection relay reliable. Judging whether the inrush current is generated by the following criteria:

$$
K=\max \left\{\frac{I_{d A 2}}{I_{d A 1}}, \frac{I_{d B 2}}{I_{d B 1}}, \frac{I_{d C 2}}{I_{d C 1}}\right\} \geq K_{s e t}
$$

where $K_{\mathrm{set}}$ is 2 nd harmonic braking ratio, $I_{\mathrm{dA} 1}, I_{\mathrm{dB} 1}, I_{\mathrm{dCl}}$ are three-phase fundamental current values, $I_{\mathrm{dA} 2}, I_{\mathrm{dB} 2}, I_{\mathrm{dC} 2}$ are three-phase 2nd harmonic current values. In the second stage when differential relay is locked by the method of second harmonic brake, the change trend of the content of second harmonic and fundamental wave are tracked. As for transformers without fault, the fundamental content of the differential current can increase in no-load input moment, and then slowly decay, so the per-unit value of differential current fundamental content variation is

$$
\Delta \dot{I}_{d \phi 1}(n)<0
$$

However, when the faulty power transformer no-load closes, the differential current in the closing moment may increase and remain the maximum value without attenuation, so there is $\Delta \dot{I}_{d \phi 1}(n) \geq 0$. In consideration of the change characteristic after no-load switching in of differential current of faulty transformer, a floating threshold value is set:

$$
\varepsilon_{\phi}(n)=\lambda \Delta \dot{I}_{d \phi 1}(n)
$$

where $\varepsilon_{\varphi}(\mathrm{n})$ is the floating threshold of sample point $n, \lambda$ is reliable coefficient.

In this case, second harmonic braking ratio becomes $K_{\text {set }}+\varepsilon_{\varphi}(\mathrm{n})$. As to power transformer operating normally, differential current decreases slowly after no-load switching in. Therefore,

$$
\varepsilon_{\phi}(n)<0, K_{\text {set }}+\varepsilon_{\phi}(n)<K_{\text {set }}
$$

That is to say, the brake ratio to judge magnetizing inrush current is decreased so that the differential relay can be reliably prevented from being affected by the fluctuation of second harmonic in the second stage. On the other hand, with regard to faulty power transformer, the differential current increases for some time and will keep a certain value,

$$
\varepsilon_{\phi}(n) \approx 0, K_{\text {set }}+\varepsilon_{\phi}(n) \approx K_{\text {set }}
$$

As is known above, the content of 2nd harmonic of differential current in fault phase is less and decays faster than in non-fault phase's, whose brake ratio is higher compared to the non-fault phase's, which ensures that 2 nd harmonic content in fault phase meets the following conditions:

$$
K_{d \phi 2}(n)<K_{s e t}+\varepsilon_{\phi}(n)
$$

As a result, in the second stage differential protection may reset three-phase differential relay with three-phase OR gate brake, and turn to the method of split phase brake, which the differential protection won't be affected by the inrush current of non-fault phase to delay action even when the faulty transformer no-load closes. Setting floating threshold, according to (6), avoids the maloperation of differential relay when the content of second harmonic changes and also contributes to quick tripping if internal fault occurred. Under the condition of no-load closing with internal fault, the properties and change characteristic of differential current and second harmonic together with some auxiliary criterion as follows can help the differential relay on fault phase make reliable action to remove fault:

$$
\begin{gathered}
I_{d \phi 1}(n) \geq I_{d \phi 1}(n-m) \\
K_{d \phi 2}(n)<K_{d \phi 2}(n-m)-\varepsilon
\end{gathered}
$$

In $(8), \varepsilon$ is determined by attenuation characteristics of inrush current of transformer.

\section{Simulation Analysis}

\section{A. Simulation Analysis of No-Load Switching-In with Transformer Internal Fault}

Simulation model of no-load switching-in with transformer internal fault is set up. Simulation lasted for 3s. Circuit breaker QF2 didn't operate. The internal fault was short circuit between A phase and B phase during 0.1 s to $3 \mathrm{~s}$. Breaker QF1 closed on the $0.2 \mathrm{~s}$. The simulation waveform is shown in Figure 1.

The differential current of $\mathrm{A}$ and $\mathrm{B}$ phase in the closing moments increased rapidly and kept the value. Due to the presence of the fault current, inrush current of fault phase was not obvious, while differential current of $\mathrm{C}$ phase had obvious characteristics of inrush current. The content of second harmonic of differential current in fault phase was less than the non-fault phase's, and decayed faster. 


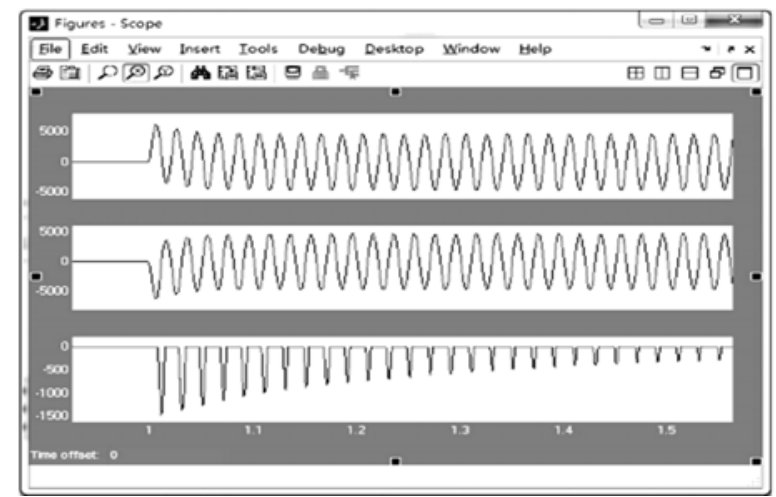

FIGURE I. SIMULATION WAVEFORM OF NO-LOAD SWITCHING-IN WITH TRANSFORMER INTERNAL FAULT

\section{B. Simulation of Magnetic Inrush Identification}

RMS value of differential current and the change characteristics of second harmonic content can be obtained by simulation of transformer when no-load closing, as shown in Table -2:

TABLE I. FUNDAMENTAL WAVE IN DIFFERENTIAL CURRENT

\begin{tabular}{|l|l|l|l|}
\hline \multirow{2}{*}{ Time/ms } & \multicolumn{3}{|c|}{ Fundamental Wave(A) } \\
\cline { 2 - 4 } & A phase & B phase & C phase \\
\hline 30 & 3217 & 3295 & 465 \\
\hline 40 & 3360 & 3394 & 428 \\
\hline 50 & 3256 & 3297 & 413 \\
\hline 60 & 3330 & 3361 & 381 \\
\hline 70 & 3297 & 3314 & 369 \\
\hline 80 & 3315 & 3343 & 340 \\
\hline
\end{tabular}

TABLE II. 2ND HARMONIC IN DIFFERENTIAL CURRENT

\begin{tabular}{|l|l|l|l|}
\hline \multirow{2}{*}{ Time/ms } & \multicolumn{3}{|c|}{ 2nd Harmonic (\%) } \\
\cline { 2 - 4 } & A phase & B phase & C phase \\
\hline 30 & 4.65 & 3.88 & 71.5 \\
\hline 40 & 4.16 & 3.62 & 71.8 \\
\hline 50 & 4.08 & 3.69 & 73.2 \\
\hline 60 & 3.72 & 3.45 & 73.5 \\
\hline 70 & 3.66 & 3.46 & 74.8 \\
\hline 80 & 3.36 & 3.22 & 75 \\
\hline
\end{tabular}

With rated current of transformer on the high voltage side $I_{\mathrm{g}}=824 \mathrm{~A}$, second harmonic braking ratio setting value $K_{\text {set }}=10 \%$, reliability coefficient $\lambda=0.8$.

According to the data in the table, if using the traditional three-phase OR gate brake, differential protection may be rejected. With adaptive second harmonic principle, $K_{\mathrm{d} 2}=69 \%>10 \%$ and inrush current is found. Therefore, enter the second stage protection judgments. Take the 2nd calculation points (40ms moments) in the table as an example to verify $\mathrm{B}$ phase. Calculate the floating threshold value of sampling point of $B$ phase at the moment $(n=2)$ : $\varepsilon_{\varphi}(\mathrm{n})=0.8 \times(3394-3295) / 824=9.6 \%$. On second calculation point, compare the content of 2 nd harmonic and the second harmonic braking ratio: $K_{\mathrm{dB} 2}(\mathrm{n})=3.62 \%<10 \%+9.6 \%$. The result of calculation meets (6). Let $\varepsilon=0.05 \%$, both $I_{\mathrm{dB} 1}(\mathrm{n})=3394>3295$ and $K_{2 \varphi}(\mathrm{n})=3.62<3.88-0.05$ are obtained, which satisfy $(7)$ and (8). At this time, open the differential relay of B phase, and trip out quickly to clear the fault after about $40 \mathrm{~ms}$ when fault occurred.

\section{CONCLUSION}

In this paper, according to the waveform characteristics of transformer inrush current, adaptive transformer inrush current identification principle based on second harmonic is proposed. This principle introduces the floating threshold value on the basis of the traditional second harmonic brake and tracks the change characteristics of the content of second harmonic and fundamental wave in real time. This method not only inherits the advantage of the original second harmonic braking method, but also helps to identify the internal fault and inrush current quickly, and improve the sensitivity of the magnetizing inrush current.

\section{REFERENCES}

[1] Xiangning LIN, Pei LIU, Shiming LIU. Ultra-saturation on state during transformer switch in with load and its influence to transformer differential protection[J]. Preceedings of the CSEE,2002, 22(3): 6-10(in Chinese).

[2] Yubo YUAN, Yuping LU, Chen LI, et al. An adaptive second harmonic restrained method using phase angle and amplitude for inrush detection[J].Proceedings of the CSEE,2006, 26(18): 19-24(in Chinese)

[3] Kulidjian A, Kasztenny B, Campbell B.New magnetizing inrush restraining algorithm for power transformer protection[C]. Developments in Power System Protection, Conference Publication, Amsterdam, The Netherlands, 2001(in Chinese)

[4] Shuyan PAN, Yuping ZHENG, Chonghao WU, et al. A new indentification component for inrush current of power transformer[J], Automation of electric Power systems, 2011, 35(19): 63-67.

[5] Yufeng HU, Deshu CHENG. A new method to identify inrush current based on sampled value[J]. proceedings of the CSEE. 2000, 20(9): 55-58(in Chinese) 